\begin{tabular}{ll}
\hline \hline MINING AND METALLURGY INSTITUTE BOR & ISSN: 2334-8836 (Štampano izdanje) \\
UDK: 622 & ISSN: 2406-1395 (Online) \\
\hline \hline
\end{tabular}

\author{
Nenad Vušović, Milica Vlahovic ${ }^{* *}$
}

\title{
PREDICTION OF SURFACE SUBSIDENCE AND DEFORMATIONS DUE TO THE UNDERGROUND COAL MINING ${ }^{* * * *}$
}

\begin{abstract}
Throughout its historical development, mining has faced the problem of moral and material responsibility due to various types of endangerment and damage to the environment. As a result of the underground coal exploitation, a movement of the rock massif above the coal seam, and changes on the terrain surface due to the process of massif stabilizing take place. The process occurs in space and time, from the moment of balance disturbance in the massif, i.e., the beginning of excavation, during excavation, and after the final excavation of deposit, when the equilibrium state is reestablished in the massif. The character and intensity of these movements and principles according to which they are performed, depend on numerous natural and mining-technological conditions, and are specific to each individual coal deposit. Deformations on the terrain surface in the sinkhole occur in the horizontal and vertical directions. Their values serve to determine the vulnerability level of individual objects on the terrain surface. On the basis of the Pataric-Stojanovic stochastic method for the predictive subsidence and deformations calculation, an original MITSOUKO program package, supported by the spatial analyses in the Geographic Information System (GIS), was designed. A case study in Sladaja village influenced by the underground exploitation in the coal mine "Rembas"- Resavica, one of the biggest Serbian coal mines, has been chosen. The data processed in the GIS provided determining the module, sense, and direction of the displacements, sinking velocity, and possible effects of subsidence on facilities.

Keywords: underground coal mining, ground surface movements, stochastic theory, GIS, spa-
\end{abstract} tial analysis

\section{INTRODUCTION}

The surface subsidence and its harmful effects on infrastructures above mining operations is a serious problem resulting from the underground coal mining in mining basins. The inevitability of consequences for objects on the terrain surface can be related to the conditionality of the mine location. Deposits of mineral raw

materials, which are often near and below populated areas or natural and technical facilities, dictate the position of underground pits and surface mines. It is truly claimed that the fight against these phenomena is the main feature of the entire mining history. The environment and surface objects can be seriously affected by the subsidence [1].

\footnotetext{
*University of Belgrade, Technical Faculty in Bor, Vojske Jugoslavije 12 Bor, Serbia, e-mail: nvusovic@tfbor.bg.ac.rs

${ }^{* *}$ University of Belgrade, Institute of Chemistry, Technology and Metallurgy, Njegoševa 12,

Belgrade, Serbia, e-mail:m.vlahovic@ihtm.bg.ac.rs,mvlahovic@tmf.bg.ac.rs

*** This work was financially supported by the Ministry of Education, Science and Technological Development of the Republic of Serbia (Grant Nos. 451-03-68/2020-14/200131 and 451-0368/2020-14/200026).
} 


\section{STOCHASTIC MODEL OF THE \\ GROUND SURFACE MOVEMENTS DUE TO THE UNDERGROUND COAL MINING}

In the zone of mining works, an impact sinkhole occurs on the terrain surface. The shape and position of the sinkhole in relation to the excavated space depend on the mining and geological conditions of excavation. When the deposit lies horizontally, the sinkhole is symmetrical with regard to the excavated space, while with steep seams it acquires an asymmetrical shape.

Damages due to the surface subsidence can be provoked by the surface slope changes, differential vertical displacements, and horizontal strains and based on their values, the vulnerability level for individual objects on the terrain surface can be estimated. Structural damages on facilities depend on the construction method and used materials; also, the chosen mining method has a great influence [2].

Case study presents a predictive calculation of the subsidence and deformations based on the stochastic method by the Pataric and Stojanovic in the MITSOUKO program package, and the GIS model using the input data from the underground coal mine "Rembas"- Resavica in Serbia.

The calculated subsidence and deformations in the analysed period have been presented graphically and described in the spatial analysis in GIS. Hence, the system created can be a useful tool to manage the subsidence data, determine its evolution, predict deformations and future environmental and social impacts, and control corrective measures. The application of the presented method supported by GIS on the chosen area enables a more automated assessment of building damage caused by the mining activity. The procedure outlined in this paper may also be satisfactorily applied in the other counties which cope with the problem of building damage risk assessment optimization [3].
Surface subsidence can be caused by the underground mining during operations or later owing to deformations in the rock mass whereby the geologic factors such as the quantity and quality of the subsoil, rock components and superficial conditions are of significant importance [4].

The developed subsidence prediction methods with different starting assumptions for deriving equations that describe the subsidence curve are: profile function methods, inluence function methods, empirical methods and numerical models. Theoretical methods try to explain a mechanism that can predict the magnitude of subsidence: profile function methods are based on the application of inluence function of the elementary excavated volume on the ground surface subsidence; influence function methods are based on the effect of extraction the infinitesimal elements of an area; empirical methods based on the results of systematic measurements in the local conditions of a coal basin; numerical models use the finite elements methods, boundary elements method, distinct elements method and finite difference methods to calculate the displacements and subsidence of ground surface [3].

Mining prophylaxy was greatly disturbed and disabled because of the modest calculation capacity and lack of automation. Computer technology and GIS enabled benefits in this field $[2,3]$.

\section{Stochastic medium theory for subsidence and deformations prediction}

Ground surface movements, caused by the underground coal mining, are very complex and therefore difficult for modeling due to the complicated behavior of the 
overlying rock mass and land profiles. Since the behavior of overlying strata is complicated due to numerous known and unknown factors that affect and conduct moving of the rock mass, the stochastic theory of ground moving was used in these models for the mine subsidence prediction.

Generally, it is easier to predict the definite subsidence than the movements caused by sequential and complicated mining processes. Therefore, the stochastic theory can be a universal method for the mine-subsidence and deformations.

Litwiniszyn (1950) proposed a stochastic subsidence model presuming the ground mass as a discontinuous medium where an element movement towards a collapsing cavity is considered as the Markovian process $[5,6]$. According to the stochastic theory, moving of the rock mass above the excavated element might happen randomly with a certain likeliness. It assumes that the rock mass can be moved from one location to another and its shape can vary under unit element mining, however its total volume remains the same. The procedure is based on the concept of stohastic process. Since its beginning, this theory has undergone numerous and constant improvements.

Liu and Liao (1965) established a stochastic method named the Stochastic Medium Theory Model (SMTM), a profile function based on the statistic medium algorithm method, for the prognosis of underground mining-induced surface movement, which is the most commonly used method in China $[7,8]$.

The Pataric-Stojanovic (1994) stochastic method applies the mathematical statistics and starts from the assumption that the massif is multi-layered, divided by a series of cracks into a large number of elements whose movements have stochastic character. Since the subsidence is plane, two coordinates are sufficient: an abscissa parallel to the layers (horizontally) and an elevation vertically with the upward direction-to determine the position of elements in the massif $[9,10]$. For the boundary transition from a discrete division to a continuous massif, the starting point is the position of elements that are defined by the coordinates, i.e.:

$$
F(x, z+h)=\frac{1}{2} \cdot[F(x-a, z)+F(x+a, z)],
$$

where $F(x, z)$ is the function of subsidence probability.

Basic formula for calculating subsidence during the inclined seams excavation is obtained:

$$
U(x, y)=U_{0} X(x, y) Y(y),
$$

where:

$$
\begin{aligned}
& X(x, y)=\frac{1}{2}\left[\Phi\left(p \frac{a+x}{\sqrt{H \cdot \operatorname{ctg} \alpha-y}}\right)+\Phi\left(p \frac{a-x}{\sqrt{H \cdot \operatorname{ctg} \alpha-y}}\right)\right] \\
& Y(y)=\frac{1}{2}\left[\Phi\left(q \frac{b+m+y}{\sqrt{H \cdot \operatorname{ctg} \alpha-y}}\right)+\Phi\left(q \frac{b-m-y}{\sqrt{H \cdot \operatorname{ctg} \alpha-y}}\right)\right]
\end{aligned}
$$

where:

$H$ - seam depth; $\alpha$ - angle of seam dip; $a$ - dimension of excavation area; parameters:

$$
p=\frac{p_{0}}{\sqrt{\sin \alpha}} ; q=(1-\lambda \sin \alpha) Q ;
$$




$$
\begin{aligned}
& b=\frac{l \cos \alpha}{1-\lambda \sin \alpha} ; m=\frac{H \lambda \cos \alpha}{1-\lambda \sin \alpha} ; \\
& \lambda=\frac{\cos \theta}{\sin (\alpha+\theta)} ; q=\frac{q_{0}}{\sqrt{\sin \alpha}} ; \\
& \theta-\text { angle of full subsidence. }
\end{aligned}
$$

The stochastic subsidence prediction enables the calculation and presentation of surface subsidence at any point in a grid with a large number of numerical data. The subsidence curve will be the same in any profile by the dip, so it is a plane subsidence:

$$
U(y)=U_{0} Y(y),
$$

where $Y(y)$ is calculated from the Eq. (1).

\section{Deformations during subsidence}

Deformations are relative changes that occur as a result of uneven subsidence or horizontal displacements on the undermined terrain. They are calculated from the diffe- rence of absolute subsidence values, or horizontal displacements of adjacent points, reduced to a length unit.

Based on their values, the vulnerability level of individual objects on the ground surface is determined. Vertical deformations are expressed through the slope changes and curvature of the terrain. Horizontal deformations-dilatations, are expressed through elongation or shortening of individual intervals between adjacent points [11].

\section{Slope}

Subsidence values can only be measured at a limited number of discretely arranged points and the slope can be calculated from this data. The slope $(N)$ represents the ratio of difference in the subsidence of adjacent points according to their distance. It is expressed in $(\mathrm{mm} / \mathrm{m})$. In fact, it is the mean slope between the tendon $A B$ of the subsidence curve and the horizontal (Figure 1):

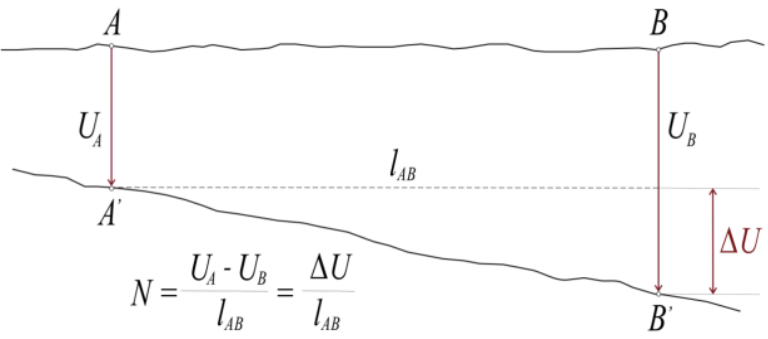

Figure 1 Slope [9]

With the coordinate origin above the middle of excavated space, the $x$-axis in a direction of coal seam dip and the $y$-axis in a direction of coal seam strike, the slopes will be positive $(+)$ by the strike and negative (-) by the dip.

If the equation $U(\xi)$ in a given profile is known, its first derivative determines the slope of tangent at some arbitrarily chosen point $M$, determined by the coordinate $\xi$ :

$$
N_{M}=N(\xi)=\frac{\partial U}{\partial \xi} \text {. }
$$

In the general case, when the equation of the subsidence curve $U(x, y)$ is known, the point $M$ and slope direction at that point must be given, because an unlimited number of vertical planes can be placed through each point on the surface of subsidence curve, whereby to each plane corresponds a different subsidence curve and thus the other slope value (Figure 2). 


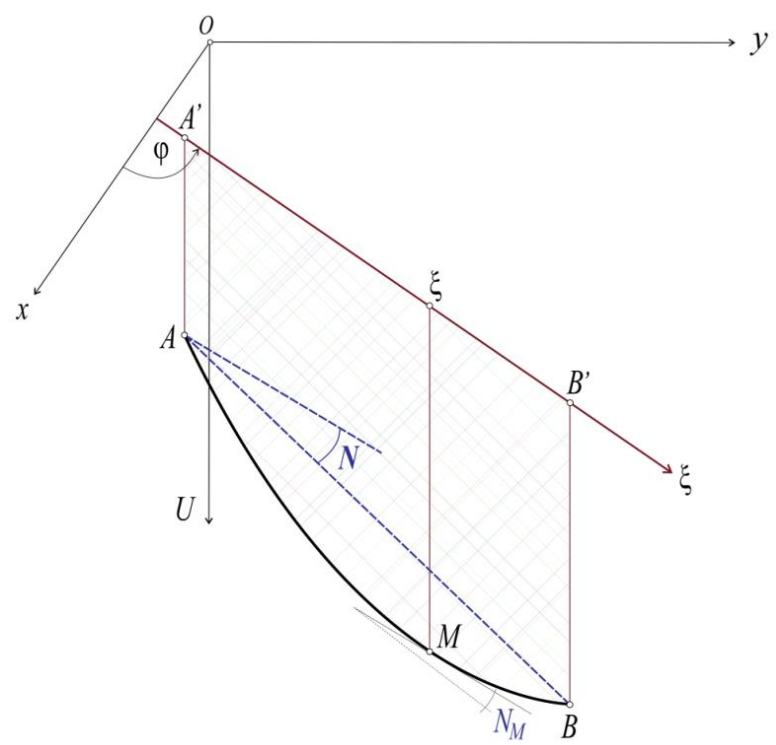

Figure 2 Slope value determination at a point $M$ on the surface of the subsidence curve [9]

To solve practical problems, it is necessary to know the main slope in the plane, in which it has the maximum value. If $\pi$ is a vertical plane through a point $M$, which makes an angle $\varphi$ with the axis $O_{x}$ (Figure 2), the coordinates of the point $M$ are:

$$
\begin{aligned}
& x_{M}=x_{A}+\xi_{M} \cdot \cos \varphi \\
& y_{M}=y_{A}+\xi_{M} \cdot \sin \varphi,
\end{aligned}
$$

then, according to Eq. 4, the slope equation will be:

$$
N_{M}(\varphi)=\frac{\partial U}{\partial x} \frac{\partial x}{\partial \xi}+\frac{\partial U}{\partial y} \frac{\partial y}{\partial \varphi},
$$

that is:

$$
\begin{gathered}
N_{M}(\varphi)=\frac{\partial U}{\partial x} \cos \varphi+\frac{\partial U}{\partial y} \sin \varphi .(5) \\
\text { If } \frac{\partial U}{\partial x}=N_{x}(x, y) \text { and } \frac{\partial U}{\partial y}=N_{y}(x, y),
\end{gathered}
$$

for a certain point $M\left(x_{M}, y_{M}\right)$ it follows that:

$$
N_{x}\left(x_{M}, y_{M}\right)=N_{x}(M)
$$

and

$$
N_{y}\left(x_{M}, y_{M}\right)=N_{y}(M) .
$$

Main slope. It can be seen from Eq. (5) that there is such a value of the angle $\varphi=\varphi_{0}$ for which the slope at an arbitrarily chosen point $M$ will be $N_{M}\left(\varphi_{0}\right)=0$, so using:

$$
N_{x}(M) \cos \varphi_{0}+N_{y}(M) \sin \varphi_{0}=0
$$

it follows that:

$$
\operatorname{tg} \varphi_{0}=-\frac{N_{x}(M)}{N_{y}(M)} .
$$

The angle $\varphi_{0}$ obviously determines a direction of subsidence contour line at the point $M$.

Also, a position of the plane $\pi$ such that the slope has an extreme value $N_{M}(\varphi)$ can 
be found; if $\varphi=\varphi_{I}$ is the angle determining

that position, from the condition

$$
\frac{N_{M}(\varphi)}{\partial_{\psi}}=0, \text { it follows that: }
$$

$$
-N_{x}(M)-\sin \varphi_{I}+N_{y}(M) \cos \varphi_{I}=0
$$

so it is:

$$
\operatorname{tg} \varphi_{I}=\frac{N_{y}(M)}{N_{x}(M)}
$$

The angle $\varphi_{I}$ determines the main direction, and the extreme value of the slope
$N_{M}\left(\varphi_{1}\right)=N_{1}(M)$ is called the main slope at the point $M$ [9].

Based on Eq. (7) for known projections $N_{x}(M)$ and $N_{y}(M)$, using familiar trigonometric identities, the following can be calculated:

$$
\begin{aligned}
& \sin \varphi_{1}=\frac{\operatorname{tg} \varphi_{I}}{\sqrt{1+\operatorname{tg}^{2} \varphi_{I}}}=\frac{N_{y}(M)}{\sqrt{N_{x}^{2}(M)+N_{y}^{2}(M)}} \\
& \cos \varphi_{1}=\frac{1}{\sqrt{1+\operatorname{tg}^{2} \varphi_{I}}}=\frac{N_{x}(M)}{\sqrt{N_{x}^{2}(M)+N_{y}^{2}(M)}} .
\end{aligned}
$$

If these results are entered in Eq. (4), the value of the main slope is obtained:

$$
N_{M}\left(\varphi_{1}\right)=N_{I}(M)
$$

$$
\frac{\partial^{2} N_{M}(\varphi)}{\partial \varphi^{2}}=-\left[\left(N_{x}(M) \cos \varphi+N_{y}(M) \sin \varphi\right)=-N_{I}(M)\right] \leq 0
$$

then this extreme value is also the maximum.

From Eqs. (6) and (7), it follows that: $\operatorname{tg} \varphi_{0} \cdot \operatorname{tg} \varphi_{I}+1=0$, so the main direction $\varphi_{I}$ is perpendicular to the subsidence contour line at the point $M$ is: $U(\mathrm{x}, \mathrm{y})=U\left(x_{M}, y_{M}\right)=$ const .

Since the main plane $\pi_{I}$ is determined by the vertical and main direction $\varphi_{I}$, it also contains the normal at the point $M$ of sinkhole, and the main slope $N_{I}(M)$ also represents the angle between the vertical

$$
N_{I}(M)=\sqrt{N_{x}^{2}(M)+N_{y}^{2}(M)} .
$$

Since the second derivative is:

and this normal. For example, it is the angle that the axis of a pillar after the massif sinking will form with its original direction (vertical).

In practice, depressions and deformations are most often determined in the main profiles, in which their extreme values also occur. However, if the excavated surface is not full, i.e., the subsidence did not reach its maximum possible value on the terrain surface, general conclusions about these values cannot be made, neither the places where the greatest deformations occur, because the result depends on the excavated field $[9,10]$. 


\section{Curvature}

Curvature represents the ratio of difference in the slope of adjacent intervals and the mean value of their lengths. It is denoted by $K$, ant its unit is $(1 / \mathrm{km})$. With slope signs, the convex curves are positive with a
(+) sign, and concave curves are negative with a (-) sign, as shown in Figure 3.

Radius of curvature is the reciprocal of curvature. It is denoted by $R$ and its unit is $(\mathrm{km})$.

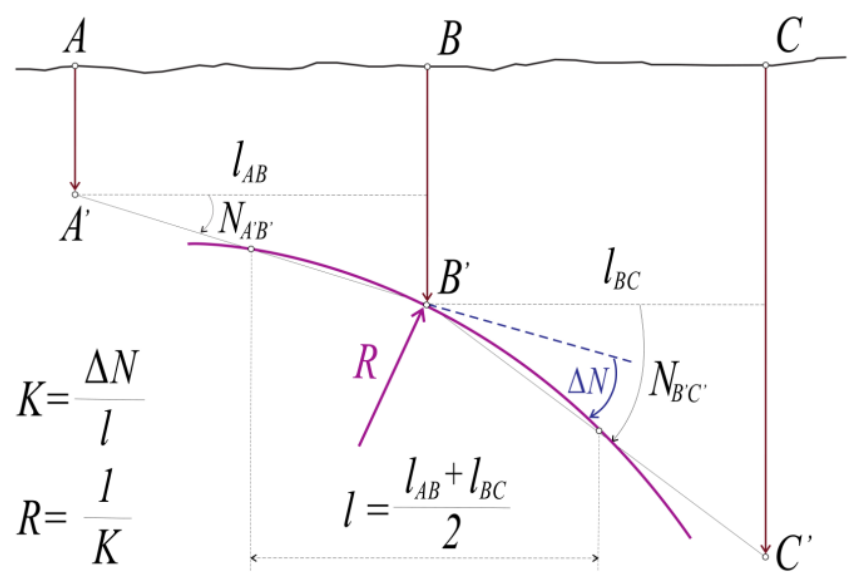

Figure 3 Curvature [9]

If the equation $U(\xi)$ of the subsidence curve in the observed profile is known, its first derivative defines the slope $N=U^{\prime}(x)$ and the second derivative determines the curvature. The curvature at any point $M$ determined by the coordinate $\xi$ can be calculated, using a known formula from differential geometry:

$$
K(\xi)=\frac{U^{\prime \prime}}{\left(1+U^{\prime 2}\right)^{3 / 2}} .
$$

As the value of the slope is small, the member $U^{\prime}={ }^{2}(\xi)=N^{2}(\xi)$ can be neglected in relation to the unity, so a sim pler formula is used to calculate the curvature at a point:

$$
K(\xi)=\frac{\partial^{2} U(\xi)}{\partial \xi^{2}}
$$

Finally, if the equation of the subsidence sinkhole $U(x, y)$ is known, a curvature can be calculated at any point $M(x, y)$ of the profile that angle $\varphi$ forms with the axis $O x$. From Figure 3, it is obvious that:

$$
\begin{aligned}
& x_{M}=x_{A}+\xi_{M} \cdot \cos \varphi \\
& y_{M}=y_{A}+\xi_{M} \cdot \sin \varphi,
\end{aligned}
$$

so, based on Eq. (12):

$$
K_{M}(\varphi)=\frac{\partial^{2} U}{\partial x^{2}}\left(\frac{\partial x}{\partial \xi}\right)^{2}+2 \frac{\partial^{2} U}{\partial x \partial y} \frac{\partial x}{\partial \xi} \frac{\partial y}{\partial \xi}+\frac{\partial^{2} U}{\partial y^{2}}\left(\frac{\partial U}{\partial \xi}\right)^{2}
$$

or, if shorter forms are introduced: 


$$
K_{x}=\frac{\partial^{2} U}{\partial x^{2}}, K_{x y}=\frac{\partial^{2} U}{\partial x \partial y}, K_{y}=\frac{\partial^{2} U}{\partial y^{2}},
$$

it follows that:

$$
K_{M}(\varphi)=K_{x} \cos ^{2} \varphi+2 K_{x y} \sin \varphi \cos \varphi+K_{y} \sin ^{2} \varphi .
$$

Instead of squares, i.e., products of sines and cosines, a double angle can be introduced, based on the known trigonometric identities:

$$
\begin{array}{ll}
\cos ^{2} \varphi=\frac{1}{2}(1+\cos 2 \varphi) ; & \begin{array}{c}
\text { So, a simpler } \\
\text { obtained: }
\end{array} \\
K_{M}(\varphi)=\frac{1}{2}\left(K_{x}+K_{y}\right)+\frac{1}{2}\left(K_{x}-K_{y}\right) \cos 2 \varphi+K_{x y} \sin 2 \varphi .
\end{array}
$$

The members $K_{\mathrm{x}}$ and $K_{\mathrm{y}}$ in this formula according to Eq. (9) represent the curvatures at the point $M(x, y)$ for profiles parallel to the coordinate axes. The third member, $K_{x y}=\frac{\partial^{2} U}{\partial x \partial y}$ represents the twist in a direction of coordinate axes at the point $M(x, y)$.

Main curvatures. To solve practical problems, it is necessary to determine the

$$
\sin ^{2} \varphi=\frac{1}{2}(1-\cos 2 \varphi)
$$

and $2 \sin \varphi \cdot \cos \varphi=\sin 2 \varphi$.

So, a simpler form of the equation is

extreme values of curvatures $K_{\mathrm{I}}$ and $K_{\mathrm{II}}$ at the point $M(x, y)$, which are called the main curvatures at a point, and the angles $\varphi_{1}$ and $\varphi_{2}$ of the corresponding profiles determine the main directions. According to Eqs. (10) and (11), the value of curvature at a point depends on the angle $\varphi$, so determiniation of extreme values of the function $K_{M}(\varphi)$ is mathematically reduced to the trigonometric equation:

$$
\frac{\partial K_{M}(\varphi)}{\partial \varphi}=-\left(K_{x}-K_{y}\right) \sin 2 \varphi+2 K_{x y} \cos 2 \varphi=0,
$$

whose solution:

$$
\tan 2 \varphi=\frac{2 K_{x y}}{K_{x}-K_{y}}
$$

determines the main directions. If the main direction is known, the other is $\varphi_{2}=90^{\circ}+\varphi_{1}$, because: $\tan 2 \varphi_{2}=\tan \left(180^{\circ}+2 \varphi_{1}\right)=\tan 2 \varphi_{1} \quad$ and therefore $\varphi_{2}$ is the solution of Eq. 13. The main directions are orthogonal.
If trigonometric identities:

$$
\begin{aligned}
& \sin 2 \varphi=\frac{\tan 2 \varphi}{\sqrt{1+\tan ^{2} 2 \varphi}}=\frac{2 \mathrm{~K}_{\mathrm{xy}}}{\sqrt{\left(\mathrm{K}_{\mathrm{x}}-\mathrm{K}_{\mathrm{y}}\right)^{2}+4 K_{x y}^{2}}} \\
& \cos 2 \varphi=\frac{1}{\sqrt{1+\tan ^{2} \varphi}}=\frac{\mathrm{K}_{\mathrm{x}}-K_{y}}{\sqrt{\left(\mathrm{K}_{\mathrm{x}}-\mathrm{K}_{\mathrm{y}}\right)^{2}+4 K_{x y}^{2}}}
\end{aligned}
$$

are entered in Eq. (11), values of the main curvatures are: 
$K_{I}=\frac{1}{2}\left(K_{x}+K_{y}\right)+\frac{1}{2} \sqrt{\left(K_{x}-K_{y}\right)^{2}+4 K_{x y}^{2}}$

$K_{I I}=\frac{1}{2}\left(K_{x}+K_{y}\right)-\frac{1}{2} \sqrt{\left(K_{x}-K_{y}\right)^{2}+4 K_{x y}^{2}}$

whereby $K_{I}$ is the highest and $K_{I I}$ the lowest value of the main curvatures $[9,10]$.

Members $K_{x}, K_{y}$ and $K_{x y}$ are calculated by the formulas:

$$
\begin{aligned}
& K_{x}=\frac{U_{0}}{n^{2}} \cdot X_{02}(x) \cdot Y_{0}(y) ; \\
& K_{y}=\frac{U_{0}}{n^{2}} \cdot X_{0}(x) \cdot Y_{02}(y)_{2} \\
& K_{x y}=\frac{U_{0}}{n^{2}} \cdot X_{01}(x) \cdot Y_{01}(y)
\end{aligned}
$$

where:

$$
\begin{aligned}
& X_{02}(x)=-\left[\frac{a+x}{n} \varphi\left(\frac{a+x}{n}\right)+\frac{a-x}{n} \varphi\left(\frac{a-x}{n}\right)\right] \\
& Y_{02}(x)=-\left[\frac{l+y}{n} \varphi\left(\frac{l+y}{n}\right)+\frac{l-y}{n} \varphi\left(\frac{l-y}{n}\right)\right] .
\end{aligned}
$$

\section{Horizontal deformations - dilatations}

Horizontal deformations- dilatations, $D$, with the unit $(\mathrm{mm} / \mathrm{m})$, are determined from the displacement of neighbouring points on the terrain surface in the horizontal plane (Figure 4) and expressed through elongation $(+)$, or shortening (-) of the interval between adjacent points (Eq. 15):

$$
\pm D=\frac{\Delta P}{l_{A B}}
$$

where: $\Delta P=P_{B}-P_{A}$

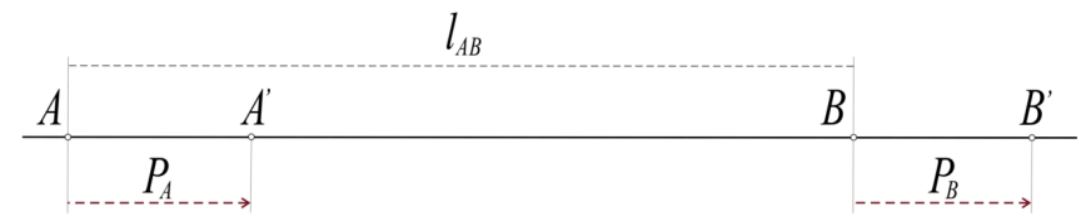

Figure 4 Horizontal deformations- dilatations [9]

Elongation occurs if, after moving the undermined terrain, the horizontal distances between adjacent points are increased, and shortenings in the opposite case. When the points on the undermined massif surface move horizontally, deformations similar to those during subsidence, also occur. Two arbitrarily chosen points, $A_{0}$ and $B_{0}$, of undisturbed massif, at a distance $A_{0} B_{0}=l_{0}$ after consolidation, move into position $A$, that is $B$, and angle $v$ between the line segments $A_{0} B_{0}$ and $A B$ (Figure 4) can be found analogously to the slope (Eq. 16):

$$
v=\frac{P_{\eta A}-P_{\eta_{B}}}{l_{0}},
$$

where $P_{\eta A}$ and $P_{\eta B}$ are the distances of the points $A$ and $B$ from the original direction; the angle $v$ is called the shear strain. 


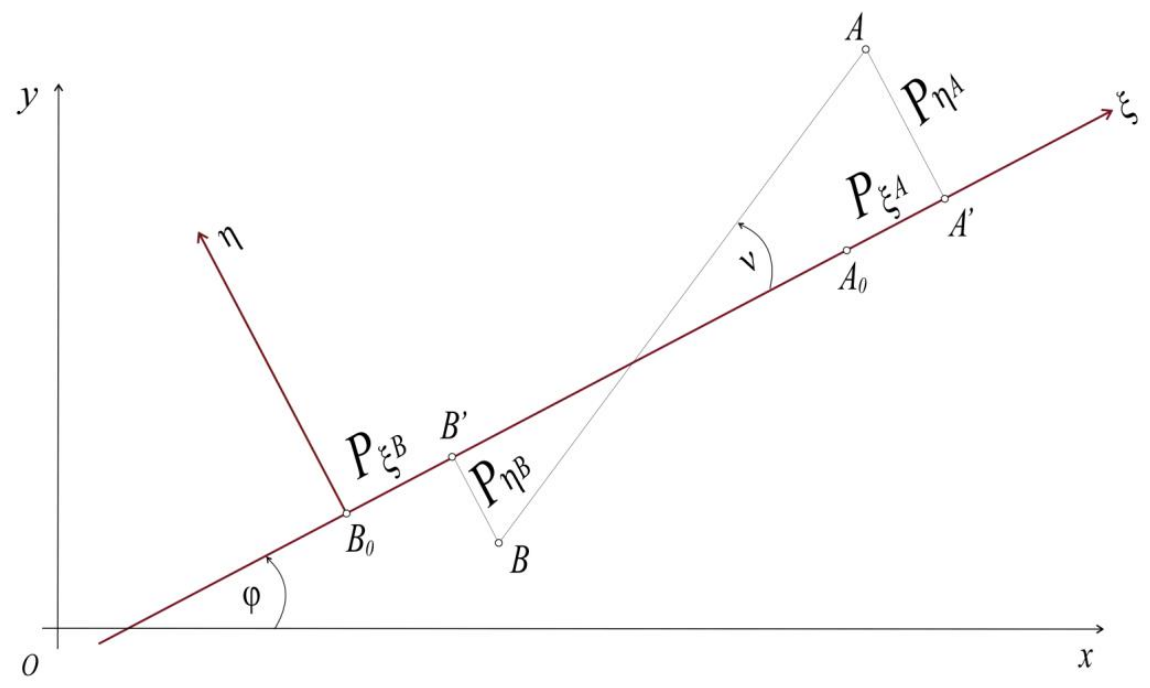

Figure 5 Shear strain [9]

The shear strain at a point $M(x, y)$ is calculated according to Eq. (17):

$v_{\mathrm{M}}=\varphi_{\mathrm{xy}}+\gamma_{\mathrm{xy}} \cos ^{2} 2 \varphi-\frac{1}{2}\left(D_{x}-D_{y}\right) \sin 2 \varphi$

whereby:

$$
\begin{aligned}
& \varphi_{\mathrm{xy}}=\frac{1}{2}\left(\frac{\partial P_{y}}{\partial x}-\frac{\partial P_{x}}{\partial y}\right), \\
& \gamma_{\mathrm{xy}}=\frac{1}{2}\left(\frac{\partial P_{y}}{\partial x}+\frac{\partial P_{x}}{\partial y}\right) \\
& D_{x}=\frac{\partial P_{x}}{\partial x}, D_{y}=\frac{\partial P_{y}}{\partial y} .
\end{aligned}
$$

where $\gamma_{x y}$ denotes slide that will be explained in the following text.

The points $A, B$ and $C$ of the undisturbed massif surface are at distances $A B=l_{1}$ and $A C=l_{2}$, and at a right angle
( $\left.A B C=90^{\circ}\right)$. Due to the rock massif undermining, there is a horizontal movement and these points move to positions $A_{1}, B_{1}$ and $C_{1}$, whereby, in general case, the angle $A_{1} B_{1} C_{1}$ is no longer a right angle. Shear strain of the line segment $(A B)$, $v_{A B}=\frac{\Delta \eta}{l_{1}}$ will be positive if $\Delta \eta>0$; the deflection $v_{A C}$ is positive if $\Delta_{\xi}>0$, so the change of right angle is $v_{A B}+v_{A C}$. Half of this change is slide:

$$
\gamma_{\xi \mu}=\frac{1}{2}\left(\frac{\Delta \eta}{l_{1}}+\frac{\Delta \xi}{l_{2}}\right)
$$

As $P_{\xi}=P_{x} \cos \varphi+P_{y} \sin \varphi$, it follows that:

$$
\begin{aligned}
& P_{\xi}=P_{x} \cos \varphi+P_{y} \sin \varphi \\
& P_{\eta}=P_{x} \sin \varphi+P_{y} \cos \varphi
\end{aligned}
$$


and:

$$
\gamma_{\xi \mu}=\gamma_{x y} \cos 2 \varphi-\frac{1}{2}\left(D_{x}-D_{y}\right) \sin 2 \varphi
$$

Half the difference $\varphi_{\xi \mu}=\frac{1}{2}\left(\frac{\Delta \eta}{l_{1}}+\frac{\Delta \xi}{l_{2}}\right) \quad$ is rotation. This quantity is not a deformation, because it does not change the shape of triangle $A B C$, but only its rotation. For the point rotation, the following can be applied:

$$
\varphi_{\xi \eta}=\varphi_{x y}=\frac{1}{2}\left(\frac{\partial P_{y}}{\partial x}-\frac{\partial P_{x}}{\partial y}\right),
$$

so the shear strain at the point is the sum of rotation and slide:

$$
v_{M}(\varphi)=\varphi_{\xi \eta}+\gamma_{\xi \eta} .
$$

Main dilatations. If $l=A^{\prime} B^{\prime}$ is the distance between the projection of points $A$ and $B$ on their original direction $A_{0} B_{0}$, according to Figure 5 , it is obvious that:

$$
\begin{aligned}
& l=\xi_{A^{\prime}}-\xi_{B^{\prime}}=l_{0}+P_{\xi_{A}}-P_{\xi B} \\
& \Delta l=l-l_{0}=P_{\xi_{A}}-P_{\xi_{B}} .
\end{aligned}
$$

In the general case $P_{\xi A}-P_{\xi B} \neq 0$, so $\Delta l$ is an elongation when $P_{\xi_{A}}>P_{\xi B}$, or a shortening when $P_{\xi_{A}}-P_{\xi_{B}}<0$.

According to Eq. (15), the relation $D_{\xi}=\frac{\Delta l}{l_{0}}$ is dilatation of line segment $A B$; it is the average elongation or the shortening of this line segment.

When the equations $P_{x}(x, y)$ and $P_{y}(x, y)$ for horizontal displacements are known, the dilatation at a point is defined analogously to the slope:

$$
D_{\xi}=\frac{\partial P_{\xi}}{\partial \xi}=\frac{\partial P_{x}}{\partial x} \cos ^{2} \varphi+\left(\frac{\partial P_{y}}{\partial x}+\frac{\partial P_{x}}{\partial y}\right) \sin \varphi \cos \varphi+\frac{\partial P_{y}}{\partial y} \sin ^{2} \varphi
$$

If denotations and a double angle are introduced, the following is obtained:

$$
D_{\xi}=\frac{1}{2}\left(D_{x}+D_{y}\right)+\frac{1}{2}\left(D_{x}-D_{y}\right) \cos 2 \varphi+\gamma_{x y} \sin 2 \varphi .
$$

This formula has the same form as Eq. (11), so the main dilatations directions are determined similarly as for curves $[9,10]$ :

$$
\tan 2 \varphi=\frac{2 \gamma_{x y}}{D_{x}-D_{y}},
$$

as well as their values:

$$
D_{I}=\frac{1}{2}\left(D_{x}+D_{y}\right)+\frac{1}{2} \sqrt{\left(D_{x}-D_{y}\right)^{2}+4 \gamma_{x y}^{2}}
$$

$$
D_{I I}=\frac{1}{2}\left(D_{x}+D_{y}\right)-\frac{1}{2} \sqrt{\left(D_{x}-D_{y}\right)^{2}+4 \gamma_{x y}^{2}} .
$$

Since the formulas have the same form as those for the curvature, it follows that the sliding in the main directions is $\gamma_{12}=0$, and the greatest sliding:

$$
\gamma_{x y}=\frac{1}{2}\left(D_{1}-D_{2}\right)
$$


is for the axes $O x$ and $O y$, which form the angle of $45^{\circ}$ with the principal axes.

\section{PROTECTION OF FACILITIES AND PROTECTION CRITERIA}

A protective pillar is a part of deposit under a facility that is not excavated or is excavated in such a way that no harmful deformations appear on the object. The endangerment of objects on the terrain surface outside the boundary of the nonexcavated protective pillar, or within its boundaries if it is excavated, depends on the type and value of deformations that may occur on the object [9].

The type of deformation according to a certain criterion depends on the object type, and the level of these deformations depends on the construction of object. There are objects, in terms of damage, sensitive only to a certain-primary type of deformation, while the other types of deformation are of secondary importance. Buildings and similar masonry buildings are most vulnerable to the horizontal deformations, which lead to the appearance of cracks. Tall buildings, chimneys, and towers are sensitive to changes in a slope. The main structures (railways, roads, pipelines) are sensitive to changes in curvature. There are objects of complex constructions that are simultaneously vulnerable to the appearance of several types of deformations, and in that case, they must be expressed through some common criterion $[2,9,12]$.

A long-term observation of behavior and damage of various facilities in different excavation conditions, empirical and statistical laws of occurrence of certain deformation types were obtained thus enabling to distinguish the limits between different intensities of damage, and to define the protection criteria. Such observations were performed in many mining developed countries with different levels of population, in different mining conditions, on facilities of different construction methods and characteristics, so that various protection criteria were created. The main difference between them is in terms of their generality, or their detail, which arises from the scope and level of study in the individual countries around the world $[12,13]$. In Serbian mining practice, there are still no adopted criteria for the protection of facilities from the impact of underground mining works [9].

According to the Polish instructions for assessing the dangerous impact of mining on buildings at the planned mining sites, which are most often used in solving these problems in the Serbian coal mines [9], the basic criteria for protection of objects are the allowed values of slope $(N)$, radius of terrain curvature $(R)$ and horizontal deformations - dilatations $(D)$. To determine the degree of dangerous impact of continuous deformations on the existing and planned facilities in the analyzed area the mining terrain is divided into categories $[2,12,14]$, whereby the limits are determined on the basis of the highest maximum values of deformations for a given category. Then the area of a given category is determined by selection the maximum ranges, which are the result of the least favorable distributions of specific deformation indices. Accordingly, the facilities are divided into four protection categories (I-IV), whereby for each category, the allowed values of deformations are prescribed, as shown in Table 1. 
Table 1 Criteria for classification the surface movements and deformations [12]

\begin{tabular}{|c|c|c|c|c|c|}
\hline \multirow[b]{2}{*}{$\begin{array}{l}\text { Terrain } \\
\text { category }\end{array}$} & \multicolumn{3}{|c|}{ Magnitude of expected deformations } & \multirow[b]{2}{*}{$\begin{array}{l}\text { Possible degree of damaging; } \\
\text { types of surface structures }\end{array}$} & \multirow{2}{*}{$\begin{array}{l}\text { Usability for } \\
\text { spatial } \\
\text { development }\end{array}$} \\
\hline & $\begin{array}{c}N_{\max } \\
(\mathrm{mm} / \mathrm{m})\end{array}$ & $\begin{array}{r}R_{\min } \\
(\mathrm{km})\end{array}$ & $\begin{array}{c}D_{\max } \\
(\mathrm{mm} / \mathrm{m})\end{array}$ & & \\
\hline $\mathbf{0}$ & $N<0.5$ & $R \geq 40$ & $D \leq 0.3$ & & \\
\hline $\mathbf{I}$ & $<0.5 N \leq 2.5$ & $40>R \geq 20$ & $0.3<D \leq 1.5$ & $\begin{array}{l}\text { Small, easily fixable damage may occur. } \\
\text { Mo-numental objects, industrial systems, } \\
\text { especially important for safety of life rea- } \\
\text { sons, or regarded as especially important, } \\
\text { e.g., gas pipelines, the damaging of which } \\
\text { may cause gas outbursts; water reservoirs. }\end{array}$ & $\begin{array}{l}\text { Safe areas, no } \\
\text { protection of ob- } \\
\text { jects is needed }\end{array}$ \\
\hline II & $<2.5 N \leq 5.0$ & $20>R \geq 12$ & $1.5<D \leq 3.0$ & $\begin{array}{l}\text { Small damage of objects may occur; rela- } \\
\text { tively easy to remove. The most important } \\
\text { objects, industrial objects, large-furnaces, } \\
\text { coke furna-ces, hoisting shafts and ma- } \\
\text { chines, Industrial objects-monolith or with } \\
\text { overhead cranes, public utility objects, e.g., } \\
\text { hospitals, theatres, vaulted churches), river } \\
\text { valleys, water reservoirs, main railways and } \\
\text { stations, tunnels, vaulted bridges, main } \\
\text { water-works unprotected against mining } \\
\text { damage, huge houses of residence (longer } \\
\text { than } 20 \mathrm{~m} \text { ). Big cities. }\end{array}$ & $\begin{array}{l}\text { Areas, where } \\
\text { partial protection } \\
\text { of all objects is } \\
\text { not profitable }\end{array}$ \\
\hline III & $5.0<N \leq 10.0$ & $12>R \geq 6$ & $3.0<D \leq 6.0$ & $\begin{array}{l}\text { Bigger damage of objects may occur, with- } \\
\text { out destroying them. The main roads, routes } \\
\text { and small railway stations, industrial objects } \\
\text { which are less susceptible to the movement } \\
\text { of the subsoil (no overhead cranes), uncoated } \\
\text { freezer-rooms, high chimneys, smaller } \\
\text { houses of residence ( } 10-20 \mathrm{~m} \text { in horizontal } \\
\text { prospection), city sewage treatment plants, } \\
\text { main collectors, sewage pipelines, gas pipe- } \\
\text { lines, steel gas pipelines. }\end{array}$ & $\begin{array}{l}\text { Areas requiring } \\
\text { partial protection } \\
\text { of objects (type of } \\
\text { protection de- } \\
\text { pends on the type } \\
\text { of object, its } \\
\text { sensitivity, subsoil } \\
\text { properties, and } \\
\text { magnitude of } \\
\text { deformations) }\end{array}$ \\
\hline IV & $10.0<N \leq 15.0$ & $6>R \geq 4$ & $6.0<D \leq 9.0$ & $\begin{array}{l}\text { Serious damage, objects are nearly de- } \\
\text { stroyed. Stadiums, small houses, other less } \\
\text { important objects. }\end{array}$ & $\begin{array}{l}\text { Areas requiring } \\
\text { serious protection } \\
\text { of objects }\end{array}$ \\
\hline $\mathbf{V}$ & $N>15.0$ & $R<4$ & $D>9.0$ & Very serious damage, objects are destroyed. & $\begin{array}{l}\text { Areas which are } \\
\text { not fit for spatial } \\
\text { development }\end{array}$ \\
\hline
\end{tabular}

\section{A CASE STUDY}

The entire raw material potential of coal in Serbia, which is predisposed to the underground exploitation systems, belongs to the JP PEU coal mines. In active coal deposits, the dominant geological forms are layered sloped structures with the pronounced tectonic deformations, whose consequences are irregular shapes of limited exploitation areas as well as possible short lengths of excavation fields and panels with frequent changes in the strike and angles of seam dip. These phenomena are the result of complex post- tectonics in the deposits. The seams from several to $40 \mathrm{~m}$ thick are from horizontal to steep. It should be added that the basic physico-mechanical properties of the working environment are relatively unfavorable because predominant are the deposits where the values of compressive strength of the mine roof and floor are lower or significantly lower than coal, which greatly narrows the possibility for application of large mechanized systems and concentration of production in them. In terms of the depth of coal 
seams, most deposits belong to the group of mines with a medium depth of exploitation, up to $500 \mathrm{~m}$. The natural-geological conditions in the deposits have a decisive role in choosing certain technological solutions of exploitation, excavation systems (methods and technologies) and security measures in the underground mining facilities [15].

\section{Characteristics of the research site}

The "Strmosten" coal deposit has a syncline shape whose wings spread to the southwest. The axis of syncline extends from the east and sinks to the west at an angle of $10-20^{\circ}$. Limestones form the rim and, for the most part, the basis of productive series, thus presenting the paleorelief of basin together with the andensites. A developed coal seam with complex structure in the "Strmosten" deposit is stratified in three parts in the northeastern area, and in two parts in the central, eastern and western areas. The thickness of coal is from 2 to $8 \mathrm{~m}$, on average $5.87 \mathrm{~m}$ at a depth of 380 to $525 \mathrm{~m}$. The barren sediments in the layer are composed of marly and coal clays, marls, clayey sandstones and sandstones. Roof deposits, with the exception of the roof immediately above the coal seam which are of the Miocene age (red sandstones, marls, sandstones, limestones and conglomerates) are most often homogeneous and undamaged. Most of the roof sandstones are red sandstones, and the conditions of collapse during excavation and level of the manifestations of pit pressures depend on their structural and physico-mechanical characteristics $[15,16$, $17,18]$.

\section{Mine subsidence prediction based on the stochastic method and spatial analysis in GIS}

The problem of surface subsidence and protecting infrastructure above the mining operations has been actual for decades in the "Strmosten" deposit. In the Technical Mining Project, the parameters of displacement the undermined terrain and protection of the facilities of the Sladaja village from the impact of underground mining works above the "Strmosten" pit were determined [17]. According to the stochastic PatarićStojanović method, subsidence at any point was calculated in the coordinate system placed in the centre of a certain excavation panel (EP). This enabled to calculate the parameters of displacement process for each $\mathrm{EP}$ and obtain complete information on the consequences of undermining on the terrain surface in the form of subsidence $(U)$ and slope $(N)$, cumulatively after successive mining of $21 \mathrm{EP}$ in the excavation field OP2 of the "Strmosten" deposit (Figure 6).

Calculating and graphical presenting the subsidence of undermined terrain is based on the originally developed software application MITSOUKO and the possibilities of spatial analysis in GIS, which are the result of modern scientific research $[3,19,20,21,22]$. Subsidence and deformations using the MITSOUKO are calculated by the simulation of excavation according to the polygonal EP and adopted mining dynamics, whereby the results were integrated and processed in GIS [3].

The basic formulas in the PatarićStojanović stochastic method, contain parameters dependent on the excavated space $(a, l, H, d, b)$ and included in the mining project, as well as parameters $\left(U_{0}, p, m, q\right)$, which are not determined 
in physical meaning, but define the behavior of the undermined terrain during consolidation and have to be brought into connection with the geometric characterristics of displacement process in the rock massif. Angles of draw $\left(\delta_{1}, \beta_{1}, \gamma_{1}\right)$,

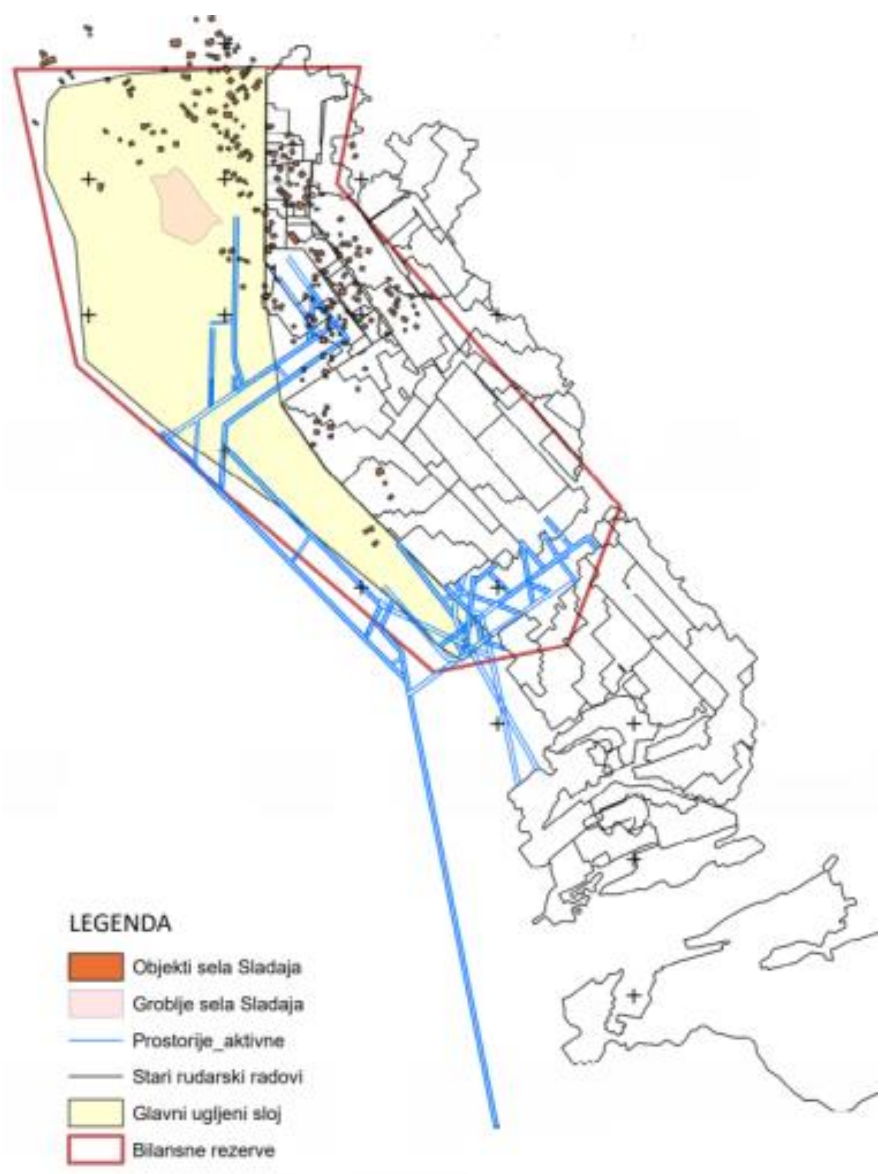

Figure 6 Excavation field OP2 of the "Strmosten " deposit [17]

which limit the zone affected by the movements on the ground surface, and angle of full subsidence $(\theta)$, as the basic angular parameters of displacement process, are among them [17]. When deriving the empirical formulas for the predictive subsidence calculation by the PatarićStojanović method, all points of the massif were observed, although the application of formulas is li-mited only to its surfaceundermined terain, since a long-term sys tematic subsidence monitoring in mines over the world were performed mainly on the surface of undermined terrain. Finally, Figure 7 presents the mining operation plan and predicted the subsidence contour lines with the maximum subsidence value ( $U_{\max }=-2927 \mathrm{~mm}$ ) after mining $21 \mathrm{ex}$ cavation panels in 2038 , that is the entire excavation field in the "Rembas" Resavica - Serbia coal mine [17]. 


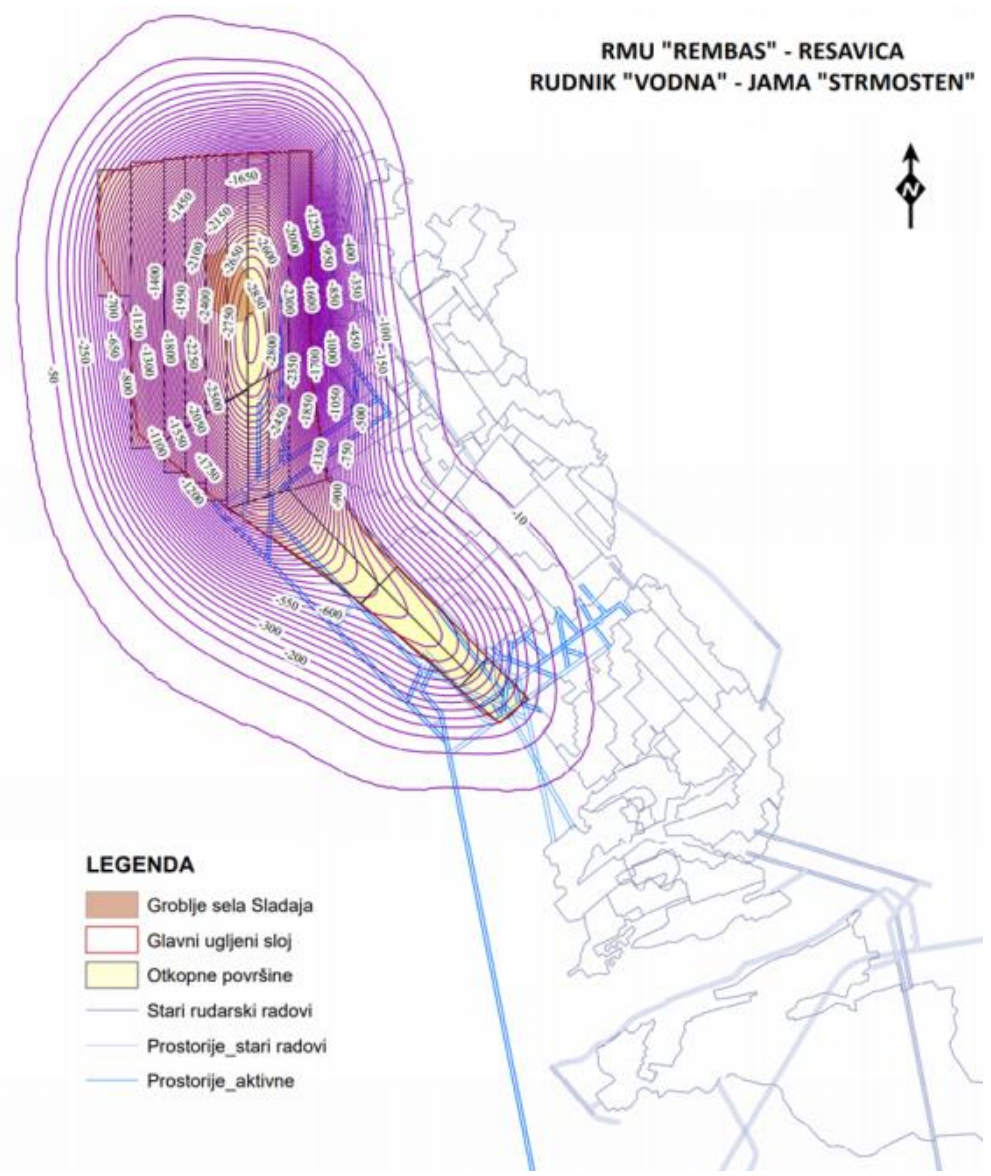

Figure 7 Subsidence contour lines after mining the entire excavation field in the coal mine "Rembas" Resavica-Serbia [17]

The MITSOUKO software, in the TILT module, calculates and tabulates the slope components at the specified points of defined EP. First, the slopes in a direction of the absolute coordinate system, formed by excavating one EP, are calculated, and then the procedure is repeated for all EPs. The components of the total slope in a direction of axes of the absolute coordinate system at a given point are obtained by summing the components of slopes of individual EPs $[3,17]$.

\section{Vulnerability Assessment of the Cemetery in the Sladaja Village}

The cemetery in the Sladaja village covers an area of $10100 \mathrm{~m}^{2}$ and a perimeter of $426 \mathrm{~m}$. Specific objects, such as monuments in the area of the cemetery in the village, are most sensitive to changes in slope $(N)$. On the other hand, this type of objects is not affected by the horizontal deformations $(D)$ and radius of curvature of the terrain $(R)$ because the dimensions of monuments are relatively small. 
To protect the terrain surface from subsidence, and thus the rural cemetery in the village, the management of the Rembas Resavica mine decided to leave a protective pillar in a part of coal seam below the sub ject area [18]. Based on the prediction [17], after excavation of all EPs without leaving a protective pillar, the calculated subsidence values in this area were from $U=-2,200$ $\mathrm{mm}$ to $U=-2,920 \mathrm{~mm}$ (Figure 8).

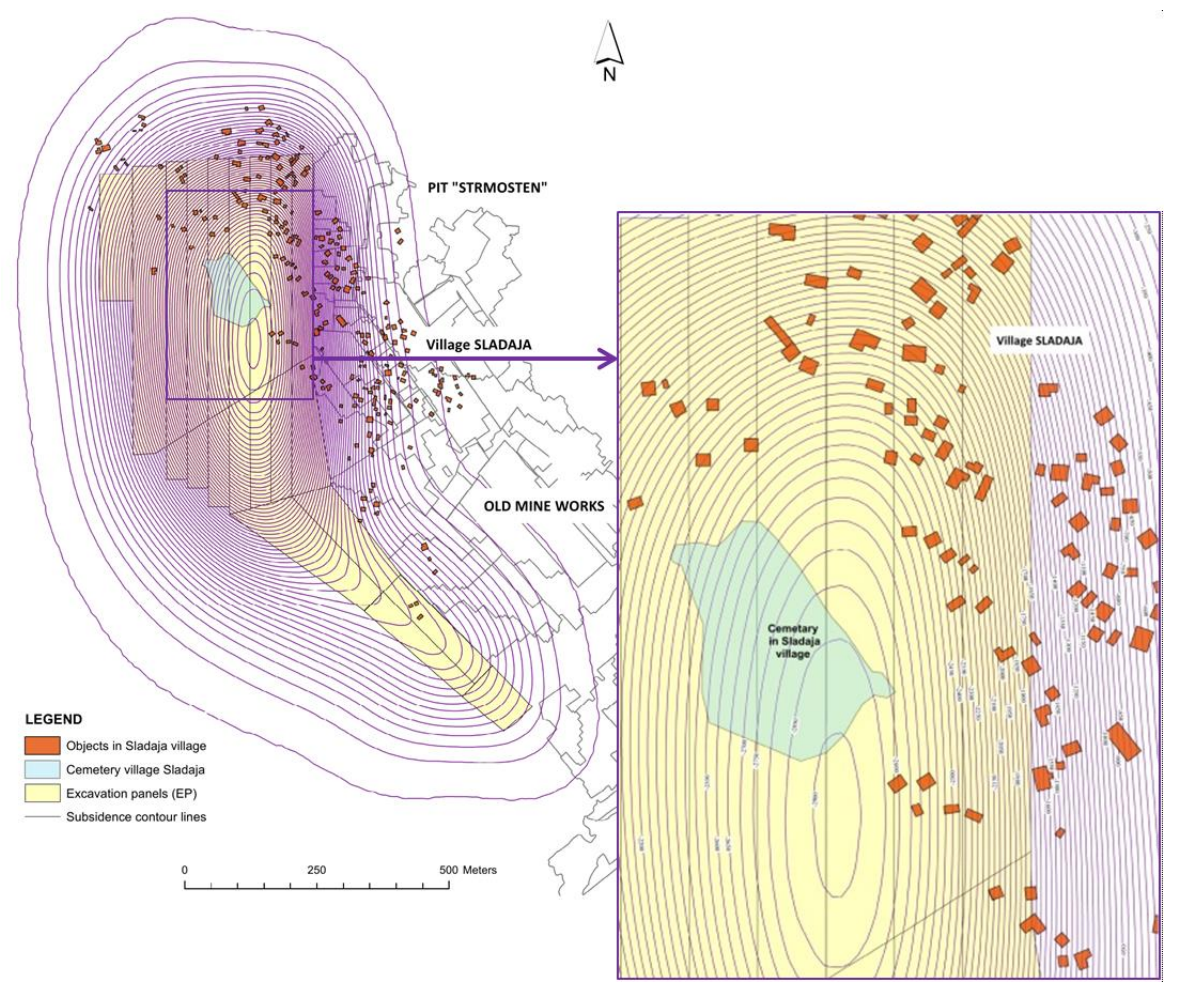

Figure 8 Subsidence contour lines after mining the entire excavation field in the surrounding of the Sladaja village and cemetery [17]

Based on these subsidence data, the slope values were calculated for the purpose of determining the deformations in the area of rural cemetery. The maximum slope was calculated at a value of $N_{\max }=$ $11.0 \mathrm{~mm} / \mathrm{m}$ in the eastern part at a very border of the cemetery; slope values $N$ in the central part of the cemetery ranged from 2 to $7 \mathrm{~mm} / \mathrm{m}$ and increased in the eastern part to a value of $14 \mathrm{~mm} / \mathrm{m}$ (Figure 9). Higher slope values in this part can be explained by the greater layer thickness $(d=6.5 \mathrm{~m})$, smaller excavation depth ( $H$ ranged from $385 \mathrm{~m}$ to $415 \mathrm{~m}$ ) and proximity of the old mining operations $[17,18]$.

By comparison the calculated slope values with the allowable values, it can be concluded that the maximum slope value of $11 \mathrm{~mm} / \mathrm{m}$ is below the allowable for objects of protection category IV (Table 1) $[12,17,18]$. 


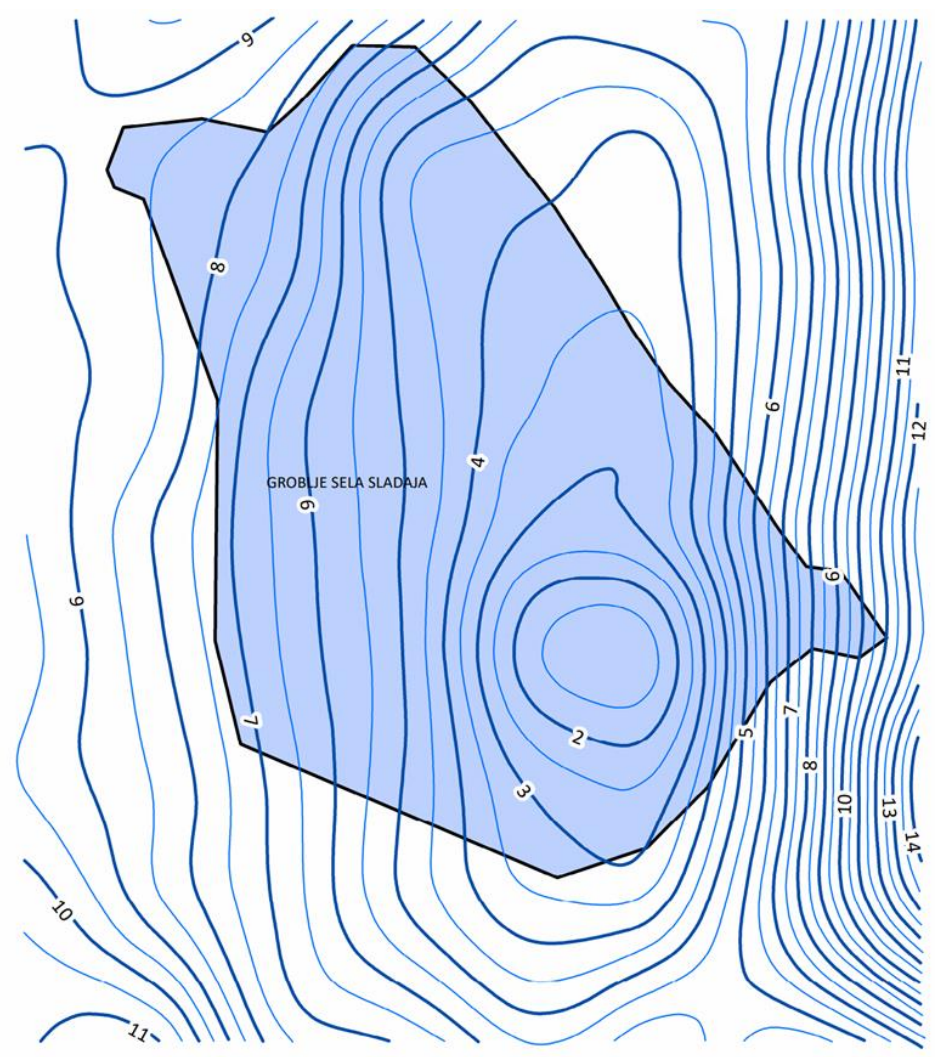

Figure 9 Slope values in the region of Sladaja village [17]

In the subsidence calculation, the deposit recovery is taken with a value of $65 \%$, which can be considered as the highest in the given conditions of mining works of the "Strmosten" pit. According to the real estimation, the excavation losses will be significantly higher, i.e., the recovery will be lower, so there is a certain uncertainty in estimation the maximum slope value on the terrain surface. It is realistic to expect that the maximum slope value will be significantly below the calculated values in the area of cemetery in the village [17].

Based on the above facts concerning the predicted values of subsidence and slope, it can be concluded that under the area of cemetery in the Sladaja village when excavating the remaining coal re- serves in the OP2 excavation field of the "Strmosten" pit in RMU "Rembas" - Resavica a protective pillar should not be left $[17,18]$.

\section{CONCLUSION}

Deformations are relative changes that occur due to an uneven subsidence or horizontal displacements on the undermined terrain. Damages owing to the surface subsidence can be the result of surface slope changes, differential vertical displacements, and horizontal strains. Based on their values, the vulnerability level for individual objects on the terrain surface is determined. Case study presents a predictive calculation of subsidence and deformations using the input data from the un- 
derground coal mine "Rembas"- Resavica in Serbia by the MITSOUKO program package, designed based on the stochastic Pataric-Stojanovic method, and supported by spatial analyses in the GIS.

\section{REFERENCES}

[1] Bell F., Stacey T., Genske D., Mining Subsidence And Its Effect on the Environment: Some Differing Examples. Environmental Geology 40 (2000) 135-152.

[2] Malinowska A., Hejmanowski R., Building Damage Risk Assessment on Mining Terrains in Poland With GIS Application, International Journal of Rock Mechanics and Mining Sciences 47 (2010) 238-245.

[3] Vušović N., Vlahović M., Ljubojev M., Kržanović D., Software Solution for The Mine Subsidence Prediction Based on the Stochastic Method Integrated With The GIS. Mining and metallurgy Engineering Bor No. 1-2 (2020) 1-16.

[4] Sanmiquel L., Bascompta M., Vintró C., Yubero T., Subsidence Management System for Underground Mining. Minerals 8(6) (2018) 243.

[5] Litwiniszyn J., The Theories and Model Research of Movements of Ground Mass, Proceedings of the European Congress in Ground Movement, Leeds, 1957.

[6] Borela V. R., Stochastic Modeling And DEM Simulation of Granular Media Subsidence Due To Underground Activity, Master Thesis, Purdue University, West Lafayette, Indiana, 2016.

[7] Liu B., Liao G., Basic Regulars of Coal Mine Subsidence, China Industry Press, 1965.

[8] Li P., Yan L., Yao D., Study of Tunnel Damage Caused by Underground Mining Deformation: Calculation, Analysis, and Reinforcement.
Advances in Civil Engineering, Article ID 4865161 (2019) 18.

[9] Patarić M., Stojanović A., Moving the Underground Terrain and Protecting Objects from Mining Works, University of Belgrade - Faculty of Mining and Geology, Belgrade, 1994.

[10] Djorđević D., Vušović N., Prognosis of the Displacement and Deformation of the Underground Terrain, University of Belgrade, Faculty of Mining and Geology, Belgrade 2014, pg. 311 .

[11] Meng F., Li-Chun W., Jia-Sheng Z., Guo-Dong D., Zhi-hui N., Ground Movement Analysis Based on Stochastic Medium Theory. Hindawi Publishing Corporation e Scientific World Journal, Article ID 702561 (2014) 1-6.

[12] Malinowska A., Hejmanowski R., Dai H., Ground Movements Modeling Applying Adjusted Influence Function. International Journal of Mining Science and Technology, 30(2) (2020) 243-249.

[13] Djamaluddin I., Mitani Y., Esaki T., Evaluation of Ground Movement and Damage to Structures from Chinese Coal Mining Using A New GIS Coupling Model. International Journal of Rock Mechanics and Mining Sciences 48(3) (2011) 380-393.

[14] Blachowski J., Application of GIS Spatial Regression Methods In Assessment of Land Subsidence In Complicated Mining Conditions: Case Study of The Walbrzych Coal Mine (SW Poland). Nat Hazards (84) (2016) 997-1014.

[15] Ivković M., Systematization of Natural-Geological Conditions of Coal Exploitation in the Underground Mines in Serbia, Monograph, Committee for Underground Exploitation of Mineral Resources Resavica. Family Press, Kragujevac, 2012 (in Serbian) 
[16] Patarić M., Supplementary Mining Project For The Exploitation Of The Main Seam at The "Strmosten" Pit, Technical Mining Project for The Excavation Impact on The Damage of Objects on The Surface. Ugaljprojekat, Belgrade 1983.

[17] Vušović N., Technical Mining Project for Determining The Parameters of Displacement of Undermined Terrain and Protection of Sladaja Village Objects From The Infuence of Underground Mining Works Above The "Strmosten" Pit at RMU "Rembas" Resavica. University of Belgrade - Technical Faculty in Bor, Bor 2018.

[18] Djukić B., Vušović N., et al., Supplementary Mining Project for Development and Excavation the Remaining Reserves in the Excavation Field 2 of the Pit "Strmosten", The Mine "Vodna", RMU "Rembas" - Resavica, Ugaljprojekat, 2019 (in Serbian)

[19] Esaki T., Djamaluddin I., Mitani Y., A GIS-based Prediction Method To Evaluate Subsidence-Induced
Damage From Coal Mining Beneath A Reservoir Kyushu, Japan. Q J Eng Geol Hydroge, 41(3) (2008) 381392.

[20] Djamaluddin I., Mitani Y., Ikemi H., GIS-Based Computational Method for Simulating the Components of 3D Dynamic Ground Subsidence during the Process of Undermining, International Journal of Geomechanics, 12(1) (2012) 43-53.

[21] Banerjee T. K., Roy S., Dey S., A GIS Solution for an Integrated Underground Coal Mine Management: A Conceptual Framework. Journal of Management Policies and Practices 2(2) (2014)129-143.

[22] Cai Y., Jiang Y., Liu B., Djamaluddin I., Computational Implementation of A GIS Developed Tool for Prediction of Dynamic Ground Movement and Deformation Due to Underground Extraction Sequence. International Journal of Coal Science \& Technology 3(4) (2016) 379-398. 